\title{
Barrierefunktion der Haut und Allergene
}

\section{Kristiane Aasen Engebretsen Jacob Pontoppidan Thyssen}

National Allergy Research Centre, Department of Dermato-Allergology, Herlev and Gentofte Hospital, University of Copenhagen, Hellerup, Denmark

\section{Zusammenfassung}

Die Haut ist eine wichtige Barriere, die den Organismus vor mechanischer Belastung, Mikroorganismen, Chemikalien und Allergenen schützt, umgekehrt aber auch die wichtige Funktion erfüllt, den Flüssigkeitsverlust zu reduzieren. Ein gemeinsames Kennzeichen vieler Dermatosen ist eine beeinträchtigte Funktionsfähigkeit der Hautbarriere, und man könnte vermuten, dass hier durch vermehrtes Eindringen potenzieller Allergene ein erhöhtes Risiko für Kontaktsensibilisierung (CS) und Allergie besteht. Die Beziehung zwischen häufigen Hauterkrankungen wie Psoriasis, atopischer Dermatitis (AD) und irritativer Kontaktdermatitis (ICD) einerseits und der Entwicklung einer Kontaktallergie (CA) andererseits ist jedoch komplex und hängt außer vom Zustand der Hautbarriere auch von verschiedenen immunologischen Reaktionen ab. Psoriasis ist in der Vergangenheit immer als Th1-dominierte Krankheit betrachtet worden, doch die Entdeckung von Th17-Zellen und IL-17 hat neue und interessante Informationen bezüglich der Pathogenese beigesteuert. Die wissenschaftliche Evidenzlage deutet auf eine inverse Beziehung zwischen Psoriasis und CA hin, die möglicherweise durch eine erhöhte Konzentration an Th17-Zellen und den dazugehörigen Zytokinen bedingt ist. Was die AD angeht, so ist eine positive Assoziation mit CS in epidemiologischen Studien etabliert worden, allerdings liegt hierfür noch keine Erklärung vor. Experimentelle Studien zeigen jedoch eine inverse Beziehung zwischen AD und CS. Die gegensätzlichen und wechselseitig antagonistischen Einflüsse von Th1- (CS) und Th2-Zellen (AD) sind als mögliche Erklärung hierfür angeführt worden. Schließlich liegen überzeugende Belege dafür vor, dass die Exposition gegenüber Reizstoffen das CS-Risiko erhöht und Patienten mit ICD somit einem erhöhten Risiko für CA unterliegen. Hautreizungen führen zur Freisetzung von IL-1 und TNF-a, was sich wiederum auf die Aktivität antigenpräsentierender Zellen auswirkt, ihre Migration zu lokalen Lymphknoten fördert und so die Wahrscheinlichkeit für eine CS und letztlich die Entwicklung einer CA erhöht.

C 2017 S. Karger GmbH, Freiburg

\section{Natürliche Hautbarrierefunktion}

Die Epidermis, insbesondere ihre oberste Schicht, das Stratum corneum (SC), schützt den Organismus vor Angriffen von Mikroorganismen sowie vor dem Eindringen von Chemikalien und Allergenen (Barriere von außen nach innen) und reduziert, was ebenso wichtig ist, zugleich den Wasserverlust durch die Haut (Barriere von innen nach außen) [1]. Die Epidermis erneuert sich kontinuierlich. Proliferierende Keratinozyten aus dem Stratum basale wandern in Richtung der Hautoberfläche durch das Stratum spinosum, wo intrazelluläre Lipide synthetisiert und in die interzelluläre Matrix sezerniert werden. Im Stratum granulosum werden wichtige Proteine produziert, darunter Filaggrin. Schließlich erreichen die Keratinozyten das SC, wo sie kollabieren und zu kernlosen Korneozyten werden, die nach einer gewissen Zeit als Hautschuppen abschilfern [2].

Die wichtige Rolle von Filaggrin besteht vermutlich darin, Keratinfilamente zu dicht gepackten Bündeln zu aggregieren und an den «cornified envelope» anzulagern, eine starre Proteinstruktur, die Korneozyten umgibt [3]. Die Lipide in der interzellulären Matrix binden kovalent an den cornified envelope. So entsteht schließlich eine robuste, vielschichtige Struktur, die vereinfacht mit Ziegelsteinen (Korneozyten und cornified envelope) und Mörtel (hydrophile Lipide) vergleichbar ist [2, 4]. Eine verminderte Produktion von Filaggrin und seinen Metaboliten führt zu Feuchtigkeitsverlust, erhöhtem pH-Wert und beeinträchtigtem UV-Schutz der Haut. Mögliche Hauptursachen eines solchen Filaggrinmangels sind Funktionsverlustmutationen im Filaggrin-Gen (FLG) sowie die Exposition gegenüber exogenen Faktoren oder entzündlichen Prozessen [3, 5-9].

Die Menge der passiv von der Hautoberfläche verdunsteten Feuchtigkeit wird als transepidermaler Wasserverlust (TEWL) bezeichnet und ist ein Marker für die Funktion der Innen-/Außenbarriere [1]. In aller Regel korreliert die Barrierefunktion von innen nach außen mit der von außen nach innen, in bestimmten Situationen ist dies jedoch nicht der Fall. Um die Außen-/Innen-

\section{KARGER}

Fax +497614520714

information@karger.com

www.karger.com

\section{(c) 2017 S. Karger GmbH, Freiburg}

Accessible online at:

www.karger.com/kkd
Kristiane Aasen Engebretsen

National Allergy Research Centre, Department of Dermato-Allergology

Herlev and Gentofte University Hospital

Kildegårdsvej 28, 2900 Hellerup, Denmark

kristiane.aasen.engebretsen.02@regionh.dk 
barriere zu evaluieren, müssen Studien zur Durchdringung mit Tracerverbindungen durchgeführt werden, was jedoch problematisch sein kann, da die Tracerverbindung typischerweise durch chemische Analyse nachgewiesen werden muss.

Eine Kontaktsensibilisierung (CS) gegenüber Chemikalien kommt häufig vor und betrifft bis zu 20\% der Allgemeinbevölkerung [10] Sie ist gekennzeichnet durch die Induktion spezifischer T-Lymphozyten-Reaktionen, hauptsächlich durch zytotoxische T-Zellen (Tc) vom Typ 1 / und T-Helferzellen (Th) vom Typ 1, aber auch Th2-Zellen können beteiligt sein [11]. Allergene (Haptene) kommen in unterschiedlicher Größe und Polarität vor und sind entweder wasser- oder fettlöslich. In Abhängigkeit von ihren Eigenschaften dringen sie auf unterschiedliche Weise in das SC ein. Das lipidreiche SC ist beständig gegenüber wasserlöslichen Haptenen; diese Substanzen können stattdessen transzellulär durch exokrine Drüsen oder Talgdrüsenfollikel eindringen. Der Hauptweg für das Eindringen von lipidlöslichen Allergenen hingegen ist jedoch typischerweise parazellulär [12]. Wenn die topische Exposition gegenüber den chemischen Allergenen wiederholt und langanhaltend erfolgt und die individuelle Schwelle übersteigt, kommt es zur CS. Diese umfasst im Wesentlichen zwei Phasen: eine Induktionsphase, in der die Person sensibilisiert wird, und eine Effektorphase, in der die allergische Kontaktdermatitis (ACD) ausgelöst wird [13]. Verbreitete Auslöser von Kontaktallergien sind Metalle, Duftstoffe und Konservierungsstoffe in Alltagsprodukten und -gegenständen wie Kosmetika oder Schmuck. Das CS-Risiko wird nicht nur dadurch bestimmt, ob die Hautbarriere intakt ist, sondern auch durch das Sensibilisierungspotenzial der Substanz; durch die Häufigkeit, die Dauer und das Ausmaß der Exposition sowie durch das Vorliegen von Okklusion oder einer lokalen Schädigung/Reizung. Die individuell unterschiedliche Anfälligkeit spielt ebenfalls eine, wenngleich untergeordnete Rolle [14].

In diesem Kapitel betrachten wir die Hauptkomponenten von drei verbreiteten Dermatosen - Psoriasis, atopische Dermatitis (AD) und irritative Kontaktdermatitis (ICD) - und gehen jeweils auf die Zusammenhänge mit Hautbarrierefunktion, Hautsensibilisierung und Entwicklung von Kontaktallergien (CA) ein.

\section{Psoriasis}

Psoriasis ist eine chronische Erkrankung der Haut und Gelenke. Die geschätzte Prävalenz liegt zwischen 0,5 und 4,6\%, je nach ethnischer Abstammung und nördlichem Breitengrad des Wohnorts [15]. Psoriatische Haut ist durch eine Verdickung der Epidermis infolge anomaler Vermehrung und beeinträchtigter Reifung der Keratinozyten gekennzeichnet; klinisch liegen abgegrenzte erythematöse Flecken oder Plaques auf der Haut vor, die mit dicken, anhaftenden, silbernen Schuppen bedeckt sind [16]. In der Vergangenheit wurden psoriatische Läsionen primär auf die Aktivität der Th1-Zellen zurückgeführt, die IFN- $\gamma$ und TNF- $\alpha$ als wichtigste proinflammatorische Mediatoren ausschütten. Die Entdeckung der Th17-Zellen und des Zytokins IL-17 haben diese Sichtweise jedoch von Grund auf verändert [17]. Erhöhte Th17Zellzahlen sind sowohl in psoriatischen Läsionen [18] als auch im
Blut von Patienten mit Psoriasis nachgewiesen worden [19], und die Menge an IL-17-mRNA korreliert signifikant mit der Krankheitsaktivität [20]. Ein wichtiges Zytokin für die Entwicklung und Erhaltung von Th17-Zellen ist IL-23, das auch für seine Rolle bei Autoimmunerkrankungen bekannt ist [21, 22]. Entsprechend sind erhöhte IL-23-Werte sowohl in psoriatischen Läsionen als auch im Serum von Patienten mit Psoriasis gemessen worden, und eine medikamentöse Behandlung zur Blockierung von IL-23 verringert den Schweregrad der Psoriasis, was für die Relevanz des Th17/IL-23-Signalwegs bei Psoriasis spricht [23].

\section{Barrierefunktion der Haut bei Psoriasis}

Die Barrierefunktion der von Läsionen betroffenen Haut bei Psoriasis ist beeinträchtigt, dementsprechend ist der TEWL im Vergleich zu gesunder Haut erhöht [24-27]. Der TEWL steht sogar in direktem Zusammenhang mit dem klinischen Schweregrad der Läsion; bei akuten Läsionen ist er stark erhöht, bei chronischen Läsionen weniger stark [1]. In einer aktuellen Studie waren die Werte für Hautfeuchtigkeit, natürliche Feuchtigkeitsfaktoren und freie Fettsäuren (FFS) in läsionaler psoriatischer Haut durchgängig niedriger als in nicht-läsionaler und gesunder Haut [24]. Andere Studien konstatierten eine Verringerung der Ceramid(CER) 1-Werte in läsionaler Haut und schwere strukturelle Veränderungen in interzellulären Lipidlamellen [25, 28].

Da Filaggrin eine wichtige Rolle bei der finalen Differenzierung der Epidermis und der Bildung der Hautbarriere spielt, liegt die Vermutung nahe, dass zwischen FLG-Mutationen und Psoriasis ein $\mathrm{Zu}$ sammenhang besteht. Mehrere Studien haben einen solchen Zusammenhang jedoch widerlegt, zumindest für Personen nordeuropäischen Ursprungs [29-34]. Dennoch ist die Filaggrin-Expression in psoriatischen Hautläsionen im Vergleich zu nicht-befallener und gesunder Haut vermindert oder sogar vollständig ausgeschaltet [29, 35-37]; vermutlich infolge der Herunterregulierung der FilaggrinExpression durch Blockade des N-Methyl-D-Aspartat-Rezeptors $[38,39]$, der Überexpression von TNF- $\alpha[40,41]$, des IFN- $\gamma$-bedingten Mangels an Caspase-14 [42] sowie des erhöhten IL-17-Spiegels [43]. In letzter Zeit ist die potenzielle Rolle einer primären Anomalie der Hautbarriere in der Ätiopathogenese der Psoriasis verstärkt in den Blickpunkt gerückt. Es gibt mehrere Hinweise, die diesen Ansatz stützen. Erstens wurde Psoriasis vor kurzem mit der Deletion von LCE3B und LCE3C im epidermalen Differenzierungskomplex auf Chromosom 1q21 in Zusammenhang gebracht, was darauf hindeutet, dass die fragliche primäre Barriereanomalie auch zu einer sekundären Immunaktivierung führen könnte [44]. Des Weiteren ist bekannt, dass psoriatische Läsionen auch durch Traumatisierung der Haut hervorgerufen werden können (Köbner-Phänomen) [45]. Okklusionsverbände können andererseits zur Besserung oder sogar Abheilung psoriatischer Läsionen führen [46]. Und schließlich wurde in einer anderen experimentellen Studie mit Tape-Stripping eine schnelle Immunaktivierung innerhalb der Epidermis erzielt, die zur Einwanderung von Entzündungszellen aus dem Blutkreislauf in die Dermis und Epidermis führte, was wiederum auf die Möglichkeit hinweist, dass primäre Ereignisse in der Epidermis vor der sekundären Immunaktivierung stattfinden [47]. 


\section{Psoriasis und Kontaktallergie}

Klinische und epidemiologische Studien belegen eindeutig eine inverse Beziehung zwischen CA und Autoimmunerkrankungen wie Typ-1-Diabetes, rheumatoider Arthritis oder entzündlichen Darmerkrankungen [48-50].

Es wird diskutiert, ob ein solcher inverser Zusammenhang auch mit Psoriasis besteht, da Epikutan-Tests bei Patienten mit Psoriasis eine CA-Gesamtprävalenz von rund 20-25\% [51-53] bzw. sogar 68\% [54] ergeben haben. Eine große epidemiologische Studie zu Psoriasis und Begleiterkrankungen ergab jedoch, dass CA bei Psoriasis-Patienten dreimal weniger häufig auftrat als in einer Kontrollgruppe mit nicht-psoriatischen Hautkrankheiten [55]. Eine inverse Beziehung zwischen CA und Psoriasis wurde auch in einer großen patienten- und populationsbasierten Studie aus Dänemark festgestellt. Die Odds Ratio (OR) für einen positiven Epikutan-Test bei Personen mit Psoriasis betrug 0,58 (95\%-Konfidenzintervall (KI) 0,49-0,68) in der Patientenstudie und 0,64 (95\%-KI 0,42-0,98) in der Populationsstudie [56]. In Übereinstimmung mit diesen Beobachtungen ergaben zwei experimentelle Studien nach Sensibilisierung mit dem starken Allergen Dinitrochlorbenzol (DNCB) eine geringere Reaktivität bei Patienten mit Psoriasis als bei gesunden Kontrollpersonen $[57,58]$. Der Grad der Sensibilisierungsfähigkeit wurde in diesen beiden Studien nicht untersucht, eine aktuelle Studie bescheinigte jedoch Patienten mit Psoriasis und Typ-1-Diabetes eine geringere Diphenylcyclopropenon-Sensibilisierungsquote im Vergleich zu gesunden Kontrollen. Nur 26\% (3/23) der Patienten mit Psoriasis und 36\% (8/22) der Patienten mit Typ-1-Diabetes wurden sensibilisiert, verglichen mit 65\% (15/23) in der Kontrollgruppe [59]. Eine niedrigere Rate der Sensibilisierung gegenüber DNCB und Nitrosodimethylamin wurde auch bei Patienten mit rheumatoider Arthritis festgestellt, wobei der Unterschied nur für letzteres signifikant war [60]. Diese Ergebnisse deuten darauf hin, dass eine verminderte Reaktivität gegenüber Allergenen ein gemeinsames Merkmal von Autoimmunerkrankungen sein könnte.

Es wurde die Hypothese aufgestellt, dass die inverse Beziehung zwischen Psoriasis und CA auf einem erhöhten epidermalen Umsatz beruhen könnte [61]. Allerdings ist auch bei anderen Autoimmunerkrankungen eine ähnliche Tendenz zu erkennen, und es erscheint wahrscheinlicher, dass diese verschiedenen Autoimmunerkrankungen ein immunologisches Milieu gemeinsam haben, das mit dem Anstoßen einer CA-Reaktion interferiert [59]. Die Rolle von Th17 in Autoimmunerkrankungen ist umfassend etabliert [17], und es wurde nachgewiesen, dass Patienten mit Autoimmunerkrankungen höhere systemische Konzentrationen Th17-assoziierter Zytokine (IL-17, IL-6, IL-21, IL-22, IL-23) aufweisen als Kontrollpersonen [19]. Dies könnte entweder die Regulierung der antigenpräsentierenden Zellen oder die Reifung der naiven T-Zellen beeinflussen und so verhindern, dass die Gedächtnis-T-Zellen gebildet werden, die für die allergische Reaktion notwendig sind [59]. Antigenpräsentierende Zellen spielen eine zentrale Rolle bei der CS, da sie Allergene an naive T-Zellen in drainierenden Lymphknoten aufbereiten, transportieren und präsentieren. Cumberbatch et al. [62] fanden heraus, dass die Funktion epidermaler antigenpräsentierender Zellen (Langerhans-Zellen) an läsionsfreien Hautstellen bei Psoriasis-Patienten im Vergleich zur Haut von Gesunden stark beeinträchtigt war. Nach Epikutan-Tests zeigten Patienten mit Psoriasis, die gegenüber Nickel sensibilisiert waren, eine typische, aber im Vergleich zu Personen ohne Psoriasis verzögerte Reaktion [63]. Während sich bei Patienten ohne Psoriasis die positiven Epikutan-Testergebnisse nach 48-72 h zeigten, war der Epikutantest bei vielen der Psoriasis-Patienten zu diesem Zeitpunkt noch negativ. Bei den Psoriasis-Patienten erreichte die Reaktion typischerweise nach 7 Tagen ihren Höhepunkt, und die Autoren stellten fest, dass die klinisch unbeteiligten Hautareale dieser Patienten ein unterschiedliches Expressionsmuster zahlreicher Gene zeigten, die am Stoffwechsel und der Zellproliferation beteiligt sind, was das verzögerte Ergebnis erklären könnte. Diese Studie deutet darauf hin, dass falsch-negative Epikutantest-Ergebnisse infolge vorzeitiger Auswertung zur geringen Prävalenz der CA bei Patienten mit Psoriasis beitragen könnten. Die inverse Beziehung zwischen Psoriasis und CA ist jedoch weiterhin nicht vollständig verstanden, und der genaue Mechanismus bleibt noch zu klären.

\section{Atopische Dermatitis}

$\mathrm{AD}$ ist eine chronische, schubförmig verlaufende entzündliche Hauterkrankung, die durch trockene Haut, Pruritus und Dermatitis gekennzeichnet ist. Akute und chronische Dermatitis treten an distinkten Lokalisationen auf und verändern sich in der Regel mit dem Alter. Die Prävalenz ist in den letzten drei Jahrzehnten dramatisch gestiegen und liegt heute bei $15-30 \%$ bei Kindern und Jugendlichen und 2-10\% bei Erwachsenen [64]. Wie auch andere atopische Erkrankungen ist AD häufig mit IgE-vermittelter Sensibilisierung gegenüber Allergenen wie Hausstaubmilben, Nahrungsmitteln (Eier, Milcheiweiß), Pollen oder Tierallergenen assoziiert, welche sich mittels Haut-Pricktest und/oder Radio-Allergo-Sorbent-Test/IgE nachweisen lässt.

Die Pathophysiologie der AD ist komplex und bisher nicht vollständig verstanden. Unzweifelhaft spielen jedoch sowohl die genetische Prädisposition als auch exogene Auslöser wichtige Rollen [65]. Immunologisch ist bei AD der Th2-Phänotyp in der akuten Phase vorherrschend, während Th1, Th17 und Th22 an der Entzündungsreaktion bei chronischen Läsionen beteiligt sind [66]. In der Vergangenheit wurden zwei verschiedene Hypothesen diskutiert. Die erste ist eine «von innen nach außen» ansetzende Theorie, der zufolge der primäre Defekt im Immunsystem angesiedelt ist und in der Folge übermäßige IgE-Sensibilisierung, Entzündungsreaktionen und sekundäre Funktionsstörungen der Hautbarriere nach sich zieht. Die zweite ist eine «von außen nach innen» gedachte Theorie, die davon ausgeht, dass der primäre Defekt sich in der Hautbarriere befindet und durch erhöhte Allergen- und Pathogen-Exposition zur sekundären überschießenden IgE-Sensibilisierung und Entzündung führt [64, 67]. 


\section{Barrierefunktion der Haut bei atopischer Dermatitis}

Bei Patienten mit AD ist die Funktion der Hautbarriere sowohl in den von Läsionen betroffenen als auch in den nicht betroffenen Hautarealen gestört - einerseits in Form von erhöhtem Feuchtigkeitsverlust (Barrierefunktion von innen nach außen) und andererseits im Hinblick auf das vermehrte Eindringen von Allergenen und Chemikalien in die Haut (von außen nach innen). Im Vergleich zu gesunden Personen ist ein doppelt so hoher TEWL in nicht-läsionaler atopischer Haut und ein vierfach erhöhter TEWL in läsionaler atopischer Haut gemessen worden [68], wobei andere Studien weniger signifikante Unterschiede ergeben haben [69, 70]. Der Grad der TEWL-Erhöhung in der nicht-läsionalen Haut korreliert außerdem mit der Schwere der AD [71].

Verschiedene mögliche Ursachen für die Beeinträchtigung der Hautbarrierefunktion bei AD sind bereits untersucht worden, von gestörter Lipidzusammensetzung über veränderte Expression von Proteinen im cornified envelope (Involucrin und Loricrin) bis hin zu einem Ungleichgewicht in den Strukturproteinen der Epidermis $[1,72]$. Auf der Hautoberfläche von Patienten mit AD liegen signifikant weniger Talgdrüsenlipide vor als auf der von Gesunden [73]. Ebenso sind in atopischer Epidermis eine geringere Gesamtlipidmenge, insbesondere in Bezug auf die Ceramide C1 und C3, sowie eine Erhöhung der FFS und Sterine nachgewiesen worden $[1,74]$. Eine klinische Studie zur Kohlenstoffkettenlänge von FFS und CER zeigte eine Reduktion der FFA- und CERKettenlängen im läsionalen und nicht-läsionalen SC der AD-Patienten im Vergleich zu Gesunden [75]. Die Veränderungen korrelierten mit einer weniger dichten Lipidorganisation und herabgesetzten Barrierefunktion der Haut und waren in läsionaler Haut stärker ausgeprägt als in nicht-läsionaler Haut. Schließlich ist für Funktionsverlustmutationen im FLG-Gen, wie sie bei rund $30 \%$ der AD-Patienten vorliegen, bekannt, dass sie mit verringerter Hautfeuchtigkeit und Barrierefunktion einhergehen und das AD-Risiko erheblich erhöhen $[64,76]$.

\section{Atopische Dermatitis und Kontaktallergie}

Atopische Haut ist klinisch durch Xerosis und intermittierende oder chronische Dermatitis gekennzeichnet; auf molekularer Ebene ist ein Mangel an Filaggrin charakteristisch. Filaggrin ist ein histidinreiches Protein, und einer aktuellen Studie zufolge bindet Nickel, das in der Natur elektrophil ist, stark an Filaggrin [77]. Außerdem wird diskutiert, dass cis-Urocaninsäure, ein Metabolit von Filaggrin, möglicherweise in der menschlichen Haut als nickelbindendes Molekül fungieren könnte [78]. Theoretisch könnte der Filaggrinmangel somit das perkutane Eindringen von Metall-Allergenen begünstigen. Bei deutschen Erwachsenen wurde eine positive Assoziation zwischen FLG-Mutationen und subjektiv angegebener Unverträglichkeit gegenüber Nickel festgestellt (OR 4,04; 95\%-KI 1,35-12,06) [79]. Eine dänische Populationsstudie bestätigte einen Zusammenhang zwischen Nickelsensibilisierung und FLG-Mutationen, allerdings lediglich für Erwachsene ohne durchstochene Ohrläppchen [80]. Des Weiteren wurde bei FLG-Mutationsträgern Nickel-ACD in jüngerem Alter und stärkere Patch-Test-Reaktivität beobachtet als bei Personen ohne die Mutation [81]. Damit einhergehend wurde eine signifikante Assoziation zwischen CS gegen Metalle und AD sowohl bei Kindern als auch bei Erwachsenen festgestellt [82-85]. Hierbei ist jedoch zu berücksichtigen, dass Epikutan-Tests mit Metallen bisweilen Probleme bereiten und verschiedene Hautreaktionen hervorrufen können, die aussehen wie echte allergische Reaktionen. Es ist untersucht worden, ob Träger einer FLG-Mutation auch einem erhöhten Risiko einer CS gegen andere Allergene außer Nickel unterliegen [86]. Eine starke Assoziation wurde festgestellt, allerdings nur bei FLG-Mutationsträgern, die laut Selbstauskunft Dermatitis hatten. Die FLG-Mutationen allein waren nicht mit einem erhöhten Sensibilisierungsrisiko verbunden. Experimentelle Studien belegen, dass die perkutane Penetration sowohl lipoals auch hydrophiler Chemikalien an klinisch normaler Haut von AD-Patienten stärker war als an der Haut gesunder Personen [87]. Außerdem stieg tendenziell die Penetrationsrate der hydrophilen Chemikalien mit zunehmender Schwere der AD an und korrelierte signifikant mit dem Gesamt-Serum-IgE. Eine erhöhte Diffusionsrate wurde außerdem für Polyethylenglykole [88] und Natriumlaurylsulfat (NLS) ermittelt [89].

Die Prävalenz der CS bei Personen mit AD könnte auch durch die erhöhte topische Allergenexposition infolge der Behandlung beeinflusst sein. Feuchtigkeitscremes und topische Wirkstoffe wie Kortikosteroide oder Calcineurin-Inhibitoren werden häufig über längere Zeiträume verordnet, um Xerosis und Inflammation atopischer Haut zu lindern. Schwedische Kinder mit mittel- bis hochgradiger AD verwendeten einer aktuellen Erhebung zufolge signifikant mehr Feuchtigkeitscremes und topische Kortikosteroide als Kinder mit geringgradiger AD [90]. Eine aktuelle Studie ergab, dass auch Produkte, die als «hypoallergen», «von Dermatologen empfohlen» oder «ohne Duftstoffe/Parabene» gekennzeichnet sind, in hohem Maße (166/187 (88,8\%) der getesteten Produkte) ein oder mehrere Kontaktallergene enthalten und viele von ihnen auch starke Allergene [91]. Insgesamt ist atopische Haut daher in hohem Maße und oft täglich einer Vielzahl an Chemikalien ausgesetzt. Von dieser erhöhten Exposition könnte man zumindest theoretisch vermuten, dass sie zu einer entsprechend erhöhten Prävalenz der CS gegenüber Chemikalien in topischen Arzneimitteln und Körperpflegeprodukten führt. Wenn die Haut bereits entzündet ist, ist kein weiteres Gefahrensignal erforderlich, um eine ACD zu befördern, wenn ein schwaches Hapten in die Haut eingeführt wird, da es in Form der Entzündung bereits vorhanden ist. Mehrere Studien haben einen Zusammenhang zwischen AD und erhöhter Prävalenz der CS gegenüber topischen Substanzen wie Kortikosteroiden, Tixocortolpivalat, Chlorhexidin und Duftstoffen festgestellt [92-97]. Eine Allgemeinbevölkerungsstudie hat ergeben, dass bei Patienten mit AD die Prävalenz der CS gegen Kontaktallergene aus topischen Produkten höher war als bei Personen ohne AD, und diese Assoziation war besonders stark, wenn FLG-Mutationen vorlagen [80]. In einer großen US-amerikanischen Studie war die Rate der CS gegen Lanolin, einen bekannten Inhaltsstoff von Kosmetika, bei AD-Patienten höher als bei Personen ohne AD [98], und eine weitere Studie ergab, dass bei Epikutan-Tests die Wahrscheinlichkeit positiver Re- 
aktionen gegen Konservierungsmittel bei AD-Patienten höher war als in der Kontrollgruppe [92]. Bei deutschen und dänischen Patienten ist $\mathrm{AD}$ auch mit einem erhöhten Risiko für multiple Kontaktallergien $(x>3)$ in Verbindung gebracht worden [99-101]. Es ist jedoch möglich, dass die bisweilen beobachtete erhöhte Prävalenz der CS bei AD-Patienten zumindest teilweise auf falschpositive Epikutan-Testergebnisse zurückzuführen ist. Eine aktuelle Studie hat gezeigt, dass die Immunantwort bei positiven Epikutan-Tests aufDuftstoffe und in geringerem Maße auf Kautschuk eine starke Verschiebung zugunsten von Th2 zeigte [102]. Akute $\mathrm{AD}$ ist ein Th2-polarisierter Prozess, und es besteht die wenngleich spekulative Möglichkeit, dass es sich bei positiven Ergebnissen von Epikutan-Tests auf Duftstoffe und Kautschuk bei ADPatienten in Wirklichkeit um akute AD handelt und nicht um echte allergische, sondern eher unspezifische Reaktionen. Hierzu ist interessant anzumerken, dass mehrere Studien bei AD-Patienten eine höhere Prävalenz von Duftstoff-CS verzeichneten als bei den jeweiligen Kontrollpersonen [94, 96, 97]. Andere Studien wiederum stellen eine Verbindung mit Duftstoff-CS infrage [103, 104]. Darüber hinaus können Metallionen, die durch Schweißdrüsen und Haarfollikel eindringen, sowie Veränderungen im pH-Wert der Haut unspezifische Entzündungen [105] ebenso hervorrufen wie Reaktionen, die keine echten allergischen Reaktionen sind, aber möglicherweise als positiv fehlinterpretiert werden, wenn der Epikutan-Test nicht fachgerecht durchgeführt wird. Reizreaktionen auf Chemikalien und Metalle kommen bei Patienten mit AD häufig vor. In einer Studie, in der 853 Hartmetallarbeiter sich Epikutan-Tests unterzogen, wurden pustulöse Patch-Test-Reaktionen auf Nickel bei AD-Patienten gefunden, die nicht gegenüber Nickel sensibilisiert waren, wenn die Pflaster auf beschädigte oder entzündete Haut platziert wurden [106]. Deutsche AD-Patienten zeigten bei frühen Untersuchungszeitpunkten im Vergleich zu Kontrollpersonen mehr unklare und irritative Reaktionen, und auch die Reaktionen auf Duftstoffe und Formaldehyd an Tag 3 waren tendenziell stärker [107].

Obwohl ein Zusammenhang zwischen AD und CS in epidemiologischen und klinischen Studien nachgewiesen ist, haben sowohl experimentelle als auch klinische Studien auch ein deutlich niedrigeres CS-Risiko bei Patienten mit AD gezeigt. Eine Studie untersuchte den Anteil jener AD-Patienten, die auf DNCB reagierten. Dabei wurde festgestellt, dass die Reaktivität von der Schwere der Erkrankung abhing. Von den Patienten mit geringgradiger $\mathrm{AD}$ reagierten $100 \%$, während es bei den Patienten mit mittlerem und hohem AD-Schweregrad nur 95\% bzw. 33\% waren [108]. Die Patienten mit geringgradiger Erkrankung reagierten wiederum deutlich schwächer auf den DNCB-Test als Kontrollpersonen ohne AD [109]. In einer weiteren experimentellen Studie wurde die Entwicklung von CS zu Rhus bei AD-Patienten und gesunden Kontrollpersonen untersucht. Die Studie ergab, dass nur 3\% der 40 AD-Patienten eine CS entwickelten, während von den 131 gesunden Kontrollpersonen 37\% sensibilisiert wurden [110]. Die Ergebnisse einer 15-jährigen prospektiven Studie stützen die dosisabhängige inverse Beziehung zwischen AD und CS, die auch in den zuvor genannten experimentellen Studien beschrieben wurde. Bei den Patienten mit schwerer AD war die CS-Prävalenz geringer als bei den Patienten mit mittelgradiger Erkrankung [95]. Unterstützend hierzu ergab auch eine aktuelle registerbasierte klinische Studie einen inversen Zusammenhang zwischen schwerer AD und CS [111]. Die Beziehung zwischen schwerer AD und CS ist jedoch strittig; andere Studien berichten genau das Gegenteil [101].

Eine mögliche Erklärung für die beobachtete inverse Korrelation zwischen AD und CS in vorwiegend experimentellen Studien liegt in den gegensätzlichen und wechselseitig antagonistischen Einflüssen von Th1- und Th2-Zellen. Die dominante Immunantwort bei CS ist eine Typ-1-Antwort mit Entwicklung von Th1und Tc1-Effektorzellen [112]. Das Krankheitsgeschehen bei AD wird hingegen hauptsächlich durch Th2-Inflammation vermittelt. Die Gedankenkette lautet daher, dass die Th1/Tc1-Reaktion unterdrückt wird und die Sensibilisierung weniger wirksam erfolgt und/oder höhere Allergenkonzentrationen für die Sensibilisierung erforderlich sind [14]. Dies wird durch eine aktuelle Studie gestützt, in der die De-novo-Sensibilisierung von Patienten mit AD gegen DNCB mit der bei gesunden Kontrollpersonen verglichen wurde. Nach Sensibilisierung gegenüber DNCB zeigte die nicht-beteiligte Haut von AD-Patienten geringere Überempfindlichkeitsreaktionen auf eine erneute Provokation nach einem Monat im Vergleich zur Haut von gesunden Kontrollpersonen [113]. Der Grad der Sensibilisierung war mit der Art der Immunantwort assoziiert, und AD-Patienten mit geringerer Sensibilisierung zeigten eine Verschiebung zugunsten der Th2-Antwort gegenüber der normalen Th1-Antwort bei den Kontrollpersonen ohne AD. Die Zusammenhänge zwischen AD und CS sind jedoch deutlich komplizierter als ein einfaches Gleichgewicht zwischen Th1- und Th2-Antworten, und weitere T-Zell-Subpopulationen wie z.B. Th17, Th22 oder regulatorische T-Zellen könnten eine wichtige Rolle spielen, ebenso wie Barrierefaktoren; dies ist jedoch bisher noch ungeklärt [114, 115]. Das Zusammenspiel zwischen AD und CS ist in Abbildung 1 zusammengefasst.

\section{Irritative Kontaktdermatitis}

Kontaktdermatitis (CD) wird unterteilt in ACD, ICD und Protein-CD. Sie ist eine der häufigsten arbeitsbedingten Krankheiten in Industriestaaten [116]. ICD ist gekennzeichnet durch lokale Entzündung nach einmaliger oder wiederholter Exposition gegenüber einem Reizstoff [117]. Dies führt zu entzündlichen und zytotoxischen Effekten, die die angeborene, nicht aber die erworbene Immunabwehr aktivieren. Die Reizquelle kann entweder chemischer (z.B. Wasch- und Reinigungsmittel, organische Lösungsmittel, Schneidöl, Desinfektionsmittel, Wasser) oder physikalischer Natur sein (mechanische Reibung, kalte und trockene Umgebung) [118]. Akute ICD entsteht bei relativ umfassender Schädigung der Haut, häufig infolge einer unbeabsichtigten Exposition gegenüber einem starken Reizstoff wie z.B. einer Industrie- oder Laborchemikalie [119]. Sie ist gekennzeichnet durch die akute Manifestation nach der Exposition, mit Entwicklung von Erythem, Ödem, Bläschen und Blasen. Eine chronische (kumula- 
Abb. 1. Vereinfachtes pathomechanistisches Zusammenspiel zwischen Kontaktsensibilisierung (CS) und atopischer Dermatitis (AD; Nachdruck/ Übersetzung mit freundlicher Genehmigung aus Thyssen et al. [14] (S. 32; Copyright 2014, John Wiley and Sons $\mathrm{A} / \mathrm{S}$ ).

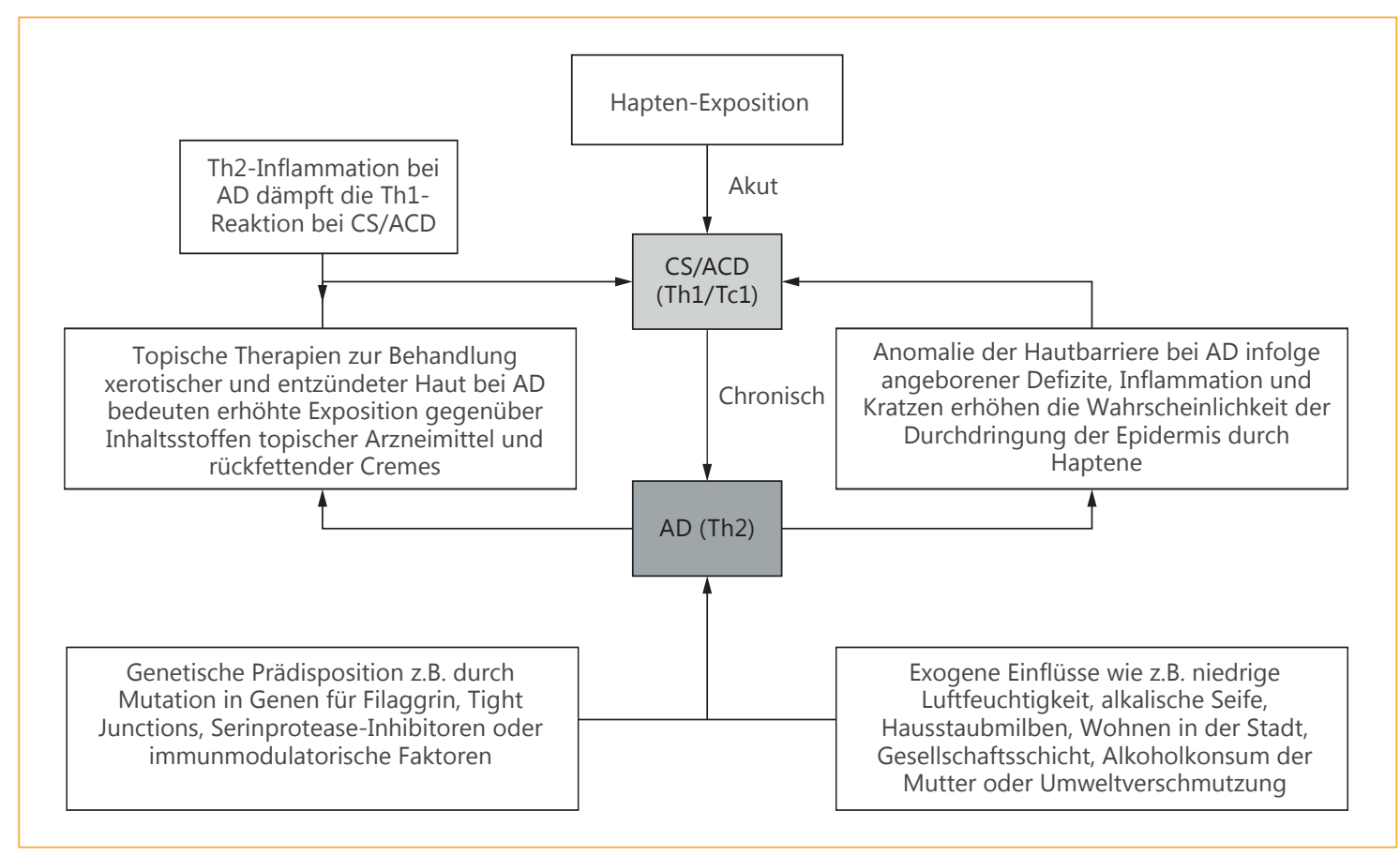

tive) ICD ist meist auf wiederholte Exposition gegenüber schwachen Reizstoffen wie Wasser und Reinigungsmittel und/oder verschiedenen physikalischen Einflüssen (Reibung, Mikrotraumatisierung, niedrige Luftfeuchtigkeit und Temperatur) zurückzuführen [117]. Durch das repetitive Auftreten der Exposition wird eine vollständige Erholung der Barrierefunktion der Haut verhindert, und das klinische Bild der Dermatitis entsteht, obwohl die einzelnen Expositionen jeweils nicht die Auslösungsschwelle überschreiten. Typische klinische Symptome sind leichtes Erythem, Xerosis, Hyperkeratose und Fissuren; sie entwickeln sich graduell über Wochen an der Kontaktfläche.

Patienten mit beeinträchtigter Hautbarriere (z.B. empfindliche Haut und $\mathrm{AD}$ ) haben eine niedrigere Reizschwelle und/oder brauchen längere Zeit zur Wiederherstellung der Hautbarriere, wodurch sie insgesamt anfälliger für ICD werden. Experimentelle und epidemiologische Daten belegen, dass eine manifeste AD oder sogar eine zurückliegende $\mathrm{AD}$ in der Kindheit das Risiko sowohl für akute als auch für chronische ICD erhöht [120-122]. Das Risiko einer chronischen ICD scheint zudem bei Personen mit FLG-Mutationen erhöht zu sein [122], auch wenn keine AD vorliegt [123].

\section{Barrierefunktion der Haut bei irritativer Kontaktdermatitis}

Anhand der TEWL-Messwerte lässt sich der Grad der Schädigung der Hautbarriere bei ICD beurteilen; die Erholungszeit nach einer Schädigung durch Reizstoffe kann über drei Wochen betragen [124]. Die Exposition gegenüber dem bekannten Hautirritans SLS verändert die Hautlipidproduktion und beeinträchtigt die Extrusion von Lipidlamellen [125]. In einer experimentellen Studie am Mausmodell führte die Durchbrechung der Barriere mit Aceton oder Tape-Stripping zur Freisetzung von Zytokinen, z.B. IL-1 $\alpha$, IL-1 $\beta$ und TNF- $\alpha$ [126]. Diese Ergebnisse suggerieren einen Zusammenhang zwischen der Exposition gegenüber Reizstoffen und Barrierestörungen und der anschließenden Aktivierung des angeborenen Immunsystems.

\section{Irritative Kontaktdermatitis und Kontaktallergie}

Der Zusammenhang zwischen ICD und CA ist sowohl in Tier- als auch in Humanstudien untersucht worden. Eine der ersten tierexperimentellen Studien, die zu Hautreizung und Hautsensibilisierung durchgeführt wurden, hat gezeigt, dass die Sensibilisierung von Meerschweinchen gegen Nickel- und Chromsalze nur unter Berücksichtigung des bekannten Irritans SLS möglich war [127]. Seitdem haben mehrere weitere Tierstudien bestätigt, dass Hautreizungen die Reaktion auf Hautallergene verstärken; die umfassendste Arbeit hierzu stammt von Magnusson und Kligman [128]. Bei Anwendung des Kontaktallergens p-Phenylendiamin bewirkt 5\% SLS einen Anstieg der Hautsensibilisierung von 38 auf 78\%; in einem anderen, ähnlichen Experiment erhöhte die Vorbehandlung mit SLS die Sensibilisierungsrate von 14 auf $46 \%$. Die Autoren gelangten zu dem Schluss, dass ein geeignetes Maß an Hautreizung Voraussetzung für eine optimale Entwicklung der Hautsensibilisierung ist. Ein weiteres deutliches Beispiel für die tiefgreifende Auswirkung von Hautreizungen auf allergische Reaktionen lieferte ein Experiment von Basketter [129], in dem SLS als Hautirritans und Isoeugenol als Kontaktallergen verwendet wurde. Meerschweinchen, die bereits gegenüber Isoeugenol sensibilisiert waren, wurden mit der maximalen nicht-irritativen Konzentration von Isoeugenol (5\%) provoziert, und 100\% der Tiere zeigten eine Reaktion. Wenn die Konzentration auf $0,05 \%$ gesenkt wurde, reagierten nur $15 \%$ der Tiere. Wenn jedoch Isoeugenol in der niedrigsten Konzentration $(0,05 \%)$ in Verbindung mit 0,05\% SLS getestet wurde (was keine klinische Erythembildung hervorruft), reagierten $90 \%$ der Tiere. 
McLelland et al. [130] führten eine der überzeugendsten Humanstudien durch. Sie wiesen nach, dass Allergendosen, die nicht hoch genug waren, um bei sensibilisierten Individuen eine allergische Reaktion auszulösen, hierzu dennoch in der Lage waren, wenn zusätzlich SLS appliziert wurde. In anderen Studien wurde der Effekt der Irritanzien-Exposition auf die Reaktionsschwelle bei Personen untersucht, die gegen Metallallergene wie Nickel, Kobalt und Chrom sensibilisiert waren. Eine 24-stündige Vorbehandlung mit 0,2\% SLS an der Epikutan-Teststelle zeigte eine deutliche Auswirkung auf die Schwellenkonzentration zur Auslösung einer (kontaktallergischen) Reaktion bei Patienten mit Kobaltallergie [131]. An der mit SLS vorbehandelten Stelle wurde eine Reaktion bereits rund eine Größenordnung früher erzielt als an den nicht behandelten Seiten. Bei Nickel konnte der Schwellenwert für die Reaktion in einer ähnlich aufgebauten Studie um eine bis zwei Größenordnungen reduziert werden [132]. Eine experimentelle Studie von Agner et al. [133] stützt diese Ergebnisse ebenfalls. Sie belegt, dass eine gleichzeitige Exposition gegenüber SLS und Nickelchlorid ( $\mathrm{NiCl} 2)$ bei Patienten mit Nickelallergie nicht nur eine additive, sondern eine synergistische Wirkung auf die Reaktion ausübte, beurteilt anhand des klinischen Befunds und der Kolorimetrie.

Die mechanistischen Hintergründe dieser Beobachtungen sind noch ungeklärt. In der Forschung wurde postuliert, dass die Allergene (Haptene) sowohl ein irritatives als auch ein antigenes Signal übermitteln und dass das erstere in der Lage ist, die Zytokinausschüttung in nicht-immunologischen Hautzellen wie z.B. Keratinozyten zu stimulieren [134]. Darüber hinaus spielen Langerhans-Zellen eine wichtige Rolle im Sensibilisierungsprozess, und ihre Fähigkeit, in lokale Lymphknoten einzuwandern, scheint von den Zytokinen IL-1 und TNF- $\alpha$ abzuhängen [135]. IL-1 wird von Langerhans-Zellen produziert und TNF- $\alpha$ in erster Linie von Keratinozyten. Hautreizungen führen zur Ausschüttung von TNF- $\alpha$, das eine Reihe von Effekten ausübt, die die Allergensensibilisierung fördern. Hier ist insbesondere die Herunterregulierung von E-Cadherin $\mathrm{zu}$ nennen, das LangerhansZellen an die Epidermis bindet, wodurch die Migration dieser
Zellen zu lokalen Lymphknoten begünstigt wird [136]. Außerdem liegen Hinweise darauf vor, dass TNF-a die Aktivität der Typ-IVKollagenase MMP-9 erhöht, was es den Langerhans-Zellen erleichtert, durch die Basalmembran der Epidermis zu gelangen [137]. Und man nimmt an, dass TNF- $\alpha$ in drainierenden Lymphknoten die Antigenpräsentation an naive T-Zellen fördert [138].

\section{Schlussfolgerung}

In diesem Kapitel sind wir auf drei häufige Dermatosen - Psoriasis, atopische Dermatitis (AD) und irritative Kontaktdermatitis (ICD) - und ihre Beziehungen zur Hautbarrierefunktion und Entwicklung von CA eingegangen. Bei allen drei Hauterkrankungen ist die Barrierefunktion der Haut beeinträchtigt, doch die Beziehung zwischen der Hauterkrankung und der Entwicklung einer CA ist komplex und hängt von verschiedenen immunologischen Reaktionen und dem Zustand der Hautbarriere ab. Im Allgemeinen deutet die wissenschaftliche Evidenzlage auf eine inverse Beziehung zwischen Psoriasis und CA hin, die möglicherweise durch die erhöhte Konzentration an Th17-Zellen und den dazugehörigen Zytokinen bedingt ist. Was die AD angeht, so deuten epidemiologische Studien auf eine positive Assoziation mit CS hin, während experimentelle Studien für eine inverse Beziehung sprechen. Die gegensätzlichen und wechselseitig antagonistischen Einflüsse von Th1- (CS) und Th2-Zellen (AD) sind als mögliche Erklärung für die Ergebnisse der experimentellen Studien angeführt worden. Schließlich liegen überzeugende Belege dafür vor, dass die Exposition gegenüber Reizstoffen das CSRisiko erhöht und Patienten mit ICD somit einem erhöhten Risiko für CA unterliegen.

\section{Dank}

Jacob Pontoppidan Thyssen und Kristiane Aasen Engebretsen werden durch unbefristete Fördermittel der Lundbeck Foundation unterstützt. Außerdem wurde die vorliegende Arbeit durch H2020 COST Action TD1206 «StanDerm» unterstützt. Die Finanzierungsquellen spielten keine Rolle bei der Erstellung des Texts.

\section{Literatur}

Literatur online unter $w w w$.karger.com/?doi=453571 